\title{
The Semantic Analysis of Ambiguity in Advertisements
}

\author{
Tian Dong ${ }^{1,}$, , Pingping Shao ${ }^{2, b}$
}

\author{
${ }^{1}$ School of Foreign Languages, North China Electric Power University, Hebei071003,China; \\ 2 School of Foreign Languages, North China Electric Power University, Hebei071003,China. \\ ayuyanxue110@163.com, b1174681119@qq.com
}

Keywords: ambiguity, advertisement, polysemy, homonymy, structural

\begin{abstract}
Advertising , as a commercial instrument, plays an essential role in people's life, especially for the merchants and consumers. A good advertisement needs to be concise, creative, impressive, and persuasive. While, ambiguity, a ubiquitous phenomenon, can satisfy the need of advertising language. This thesis mainly analyzes the ambiguity in advertisements and aims to find out the functions of ambiguity in advertisements. The results show that ambiguity can make the advertisements concise, impressive, attractive, and persuasive.
\end{abstract}

\section{Introduction}

With the rapid development of the society,advertising has entered every corner of the world today. It plays an essential role in people's daily life. For the importance of advertising, many scholars have studied it from different perspectives. For example, the Chinese scholar Jiang Hua has talked about the advertising language and rhetorical devices in his thesis. Another scholar Kong Xingyan has done a research on the translation of advertisements from the perspective of language and culture. Also, the scholar Kong Kaichao studies advertisements language from the perspective of memetic theory.

Many scholars have studied advertisements from different perspectives, but the researches on ambiguity in advertisements have been hardly touched. Ambiguity refers that one linguistic expression denotes two or more ideas. Many scholars have done researches on ambiguity in different texts, like literary texts, court debate, and humorous occasions, etc. However, rare researches involve ambiguity in advertisements. So, this thesis focus on the analysis of ambiguity in advertisements.

As a commercial instrument, advertising is " for purpose of immediate action or to make us more favorably disposed in general terms to the advertised product or service” (Goddard, 1998:11 ). To reach this goal, it employs many techniques, like sound, illustration,pictures and so on, among which language occupies a crucial position. Advertising language is generally concise, creative, impressive and persuasive. The ambiguity, to some degree, can meet these demands of advertisements. This thesis attempts to analyze the ambiguity in English advertisements. It mainly discusses how the ambiguity rises and the functions of ambiguity in advertisements. Through the analysis, it can help consumers understand advertisements correctly and contribute to the output of effective and humorous advertisements.

\section{Theoretical framework}

\subsection{The definition of ambiguity}

Ambiguity is a common phenomenon in natural language. Many scholars of different linguistic fields have done researches of ambiguity. However, it is difficult to give an accurate definition of ambiguity. So, many scholars have tried to give a definition from their own views.

According to Webster's Third International Dictionary, ambiguity refers to the condition of admitting of two or more meanings, of being understood in more than one way, or referring to two or more things at the same time. Leech defines ambiguity from the semantic perspective, "a sentence with two or more propositions"(1981:112). Chomsky interprets ambiguity through the analysis of the deep structure and the surface structure of an expression, which comes from his famous Transformational Grammar (1965:14). And Kempson thinks that a sentence would be ambiguous if it 
could be true in quite distinctive circumstances (1977). While, in 1975, Paul Grice brings the study of ambiguity to pragmatics. His cooperative principle tells us to avoid ambiguity in case of conversational implicature.

In China, Zhao Yuanren, the famous linguist holds that ambiguity occurs when a word, phrase, or sentence has more than one meaning, and therefore should be avoided in language use in case of misunderstanding. In 2002, Xiang defines ambiguity as "a phenomenon that a certain utterance may lead to different understandings and express several illocutionary acts or forces” from pragmatic view.

As is shown above, ambiguity has been defined by many scholars in various fields. Though they are not the same, there is something in common. Ambiguity happens due to the lack of one-to-one correspondence between expressions and meanings.

\subsection{The classification of ambiguity}

The classification of ambiguity can be analyzed from different perspectives. And there is no definite standard now. From the perspective of style, it can be classified into oral ambiguity and written ambiguity. From the perspective of communicative effect, there are intentional ambiguity and unintentional ambiguity. Also, some scholars classify from the reasons causing ambiguity, that is, phonetic ambiguity, lexical ambiguity,structural ambiguity and pragmatic ambiguity. The last classification is the most popular and common one. In this thesis,the lexical ambiguity and structural ambiguity will be used, so they will be illustrated in detail.

\subsubsection{Lexical ambiguity}

The language in the world are natural, thus ambiguity is inevitable. Many new words spring up and at the same time the meaning of words is changing over the time. Some lose their old-fashioned meaning, and some acquire new meanings. The result is that polysemy and homonymy abound in every language. The result is that their presence frequently leads to ambiguity.

"Polysemy deals with multiple senses of the same phonological word." (Saeed, 64) Sometimes, the different meanings of the same polysemous word make sense. Thus, ambiguity emerges. For example, "She didn't take his tip". Here, in this example, the sentence can be understood in two ways. Firstly, "She didn’t accept his advice.” Secondly, “ She didn't accept his gratuity”. The ambiguity results from the word "tip". "Tip" may refer to advice or extra money, both of which can be comprehended in the sentence. So ambiguity comes into being.

Not only polysemy generates ambiguity, but also homonymy produces ambiguity. "Homonyms are unrelated senses of the same phonological word " . (Saeed, 63) Here, it's necessary to mention the distinction of polysemy and homonymy. Both of them deal with multiple senses of the same phonological word, but polysemy is invoked if the senses are judged to be related, while the other is judged to be unrelated. The "relatedness" is used to identify them. Specifically, homonyms are words that have the same spelling or pronunciation but different meanings (like ball, port, bank, etc). These words can also produce ambiguity. For example, "He was attracted by the ball." In this example, the ambiguity is generated due to the word "ball". Here, "ball" can be a round object or a dance party. Certainly, the two meanings are unrelated with each other.

\subsubsection{Structural ambiguity}

Grammatical structure may also lead to ambiguity. Different interpretations of a structure lead to different understandings.Structural ambiguity varies in form. But, ultimately, they are caused by the unclearness of the modification of a certain word. For example, "He came to tell me you had been to see him after tea." In this example, the prepositional phrase "after tea” produces ambiguity. Because it can either modify the predicate verb "came", or the infinitive "to see". Thus, two meanings arise. "He came after tea to tell me that you had been to see him" or "He came to tell me that after tea you had been to see him". 


\section{The analysis of ambiguity in advertisements}

Ambiguity is ubiquitous in advertisements. In this chapter, lexical ambiguity and structural ambiguity in advertisements will be analyzed in detail to explore the functions of ambiguity in advertisements and the reason why ambiguity is so popular in advertising.

\subsection{Lexical ambiguity in advertisements}

Lexical ambiguity refers to the ambiguity which arises due to the different understanding of the same word. Here, polysemy nd homonymy are two types which often appear in advertisements and lead to ambiguity. In the following part, they're presented in detail.

\subsubsection{Lexical ambiguity caused by polysemy}

If a word has several different meanings, and also there is some relatedness between these senses, then the word is called polysemantic word. (Saeed, 64) All of the meanings make sense in the same sentence, then ambiguity arises. The examples are as follows :

(1) The world's most civilized spirit.

(2) Before you check in, check out.

In example (1), it is an advertisement for Hennessy, a kind of wine. Here, this advertisement is ambiguous due to the word "spirit", which is a polysemantic word. For one thing, it refers to a kind of strong wine. For another thing, it refers to a kind of thinking activity. So, the sentence can be understood in two ways. "Hennessy is a kind of strong wine in the world". Here, it tells the consumers about the good quality of the wine, and persuades them to buy it. Also, it can be understood like this "Hennessy is the world's most civilized wine". It comes to the spiritual level. Though the wine is strong, it's not inelegant. It's appropriate for noble persons. That is to say, consumers who buy this kind of wine are very likely noble and elegant. It's a symbol of social status. The ambiguity in this advertisement enriches the information about Hennessy with simple words and stimulates consumers' desire.

In example (2), the advertisement is for a hotel. The ambiguity arises because of the verbal phrase "check out", which is polysemantic. It has two meanings. One is "arrive and register". The other is "leave a restaurant and settle accounts". So the meanings of the sentence : "Before you check in, please register at the reception desk". This is a kind of remind to the passengers. The other one is that "Before you check in, leave and settle accounts". Certainly, the second meaning is not what the advertisement really wants to express. It just wants to attract the passengers' attention. When passengers see this, they will wonder they have not entered the restaurant, why they give them money. They may be full of curiosity and enter the hotel. Thus, the advertisement takes effect.

\subsubsection{Lexical ambiguity caused by homonymy}

"Homonyms are unrelated senses of the same phonological words". (Saeed, 63) Specifically, homonyms are words that have the same spelling or pronunciation but different meanings. These words can also produce ambiguity. The examples are as follow:

(3) I'm More satisfied.

Example (3) is an advertisement for the cigarette. In this example, the word "More" has two meanings. One refers to the brand of the cigarette "More". The other refers to an adverb, expressing the amount or the degree. So, this advertisement tells consumers two pieces of information. It tells readers the brand name of the product. Also, it leaves an expression on the consumers : this kind of cigarette is superior to the others, and it makes consumers more satisfied. So, this kind of ambiguity makes consumers remember this brand name as well as persuades them to buy it.

\subsection{Structural ambiguity in advertisement}

Structural ambiguity is the ambiguity caused by the unclearness of the modification of a certain word.(Zhang ,67) So different interpretations of a structure lead to different understandings. The examples are as follow.

(4) Learn to drive fast.

(5) After you get married, kiss your wife in places she's never been kissed before.

Example (4) is an advertisement for a driving school. As far as the structure is concerned, "fast" can modify both "learn" and "drive", which causes the structural ambiguity. Therefore, it can be 
understood in two ways : "The school can teach you how to learn fast to drive", and "The school can teach you how to drive at a high speed”. Whichever meaning comes to the consumers, it will be easily understood. Also, the two interpretations will attract them. If consumers can learn fast,they will get their driving license earlier. Certainly, driving fast is also tempting for them. The advertiser deliberately employs such an ambiguous sentence to enriches information as well as to attract the driving student.

Example (5) is an advertisement for Four Corners Honeymoon Holiday. At a glance of it, two interpretations may come to consumers' mind. "After you get married, kiss your wife on her body where she's never been kissed before”. This interpretation intends to be most people's first choice for this advertisement due to high frequency of intimate words. The other interpretation may be "After you get married, kiss your wife in locations where she's never been kissed before”. This one is surly the adviser's intended objective. That is, attract consumers to come here to take their holiday.

\section{Summary}

Advertising plays an essential role in people’s daily life. A good advertisement needs to be concise, creative, impressive, and persuasive. While, ambiguity can meet the characteristics of advertising language to some degree. This thesis mainly reviews the definitions and classification of ambiguity, and analyzes the ambiguity in advertisements, including lexical ambiguity and structural ambiguity.

According to the analysis, it can be found that ambiguity plays a great role in advertisements. Firstly, it can enrich the information of advertisements with concise language. The same word may have different understandings, which all make sense. Thus, through one word, different information is delivered. Secondly, ambiguity may make the advertisements impressive. Sometimes, advertisers utilize ambiguity to create humorous effects, which will give a deep impression on consumers. Thirdly, ambiguity may make the advertisements attractive and persuasive. Sometimes, the advertisers utilize the ambiguity to improve the reputation of the products, grab people's attention, arouse their interest, and ultimately persuade them to buy the advertised products. All in all, ambiguity is a good choice for advertising due to its simplicity, rich embodiment, catchy flavor and forceful persuasion. Through this analysis, it helps consumers understand advertisements in a proper way, and contributes to the output of effective and humorous advertisements. Certainly, it will provide a new perspective of studying advertisements.

In brief, ambiguity is essential for advertisements production and comprehension. It's worth everyone's attention and needs further research. To analyze the advertisements from the perspective of ambiguity provides a new way for appreciating advertisements.

\section{References}

[1] A. Goddard, The language of advertising: written texts. [M]. London; New York: Routledge. 1998:11. <http://ishare.iask.sina.com.cn/f/20997622.html > April 20, 2012.

[2] G. Leech, Semantics:The Study of Meaning, 2nd [M]. Penguin Language and Linguistics. 1981:112.

[3] A.N.Chomsky, Syntactic Grammar. [M]. 1965:14.

[4] R. Kempson, Semantics theory [M]. Oxford: Cambridge University Press. 1977.

[5] Y.R. Zhao, Z. J. Wu, X.N.Zhao, The Ambiguity in Chinese.<Zhao Yuanren' language memoir>.[M]. Beijing commercial press, 2006: 654.

[6] C.D. Xiang, The Pragmatic Study of Ambiguity [J]. Foreign Language Teaching, 2002.

[7] J. Saeed, Semantics Beijing : Foreign language teaching and research press,2000.8.

[8] F.F. Zhang, “The study of the pragmatic functions of ambiguity in English advertising”, Foreign Language World, 2012. 